\title{
SANDIA REPORT
}

SAND99-2692

Unlimited Release

Printed Ocbober 1999

\section{RECEIVEO}

nov 02 seg

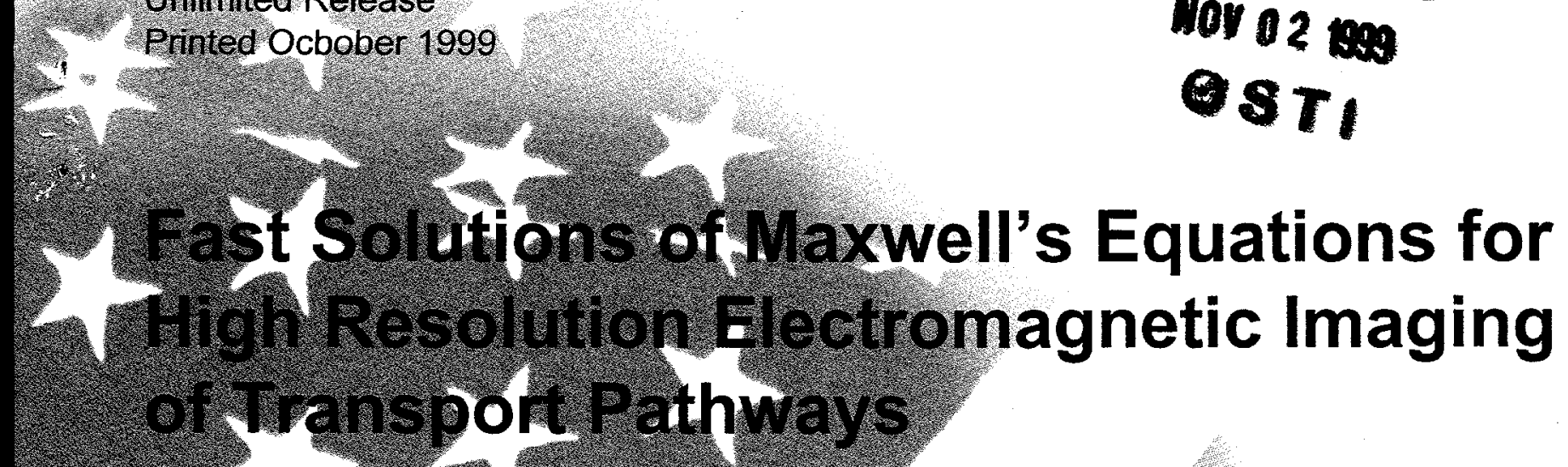

Gregory A. Newman and David M. Day

Prepared by

Sandia National Laboratories

Albuquerque, New Mexico 87185 and Livermore, California 94550

Sandia is a multiprogram laboratory operated by Sandia Corporation,

a Lockheed Martio Company, for the United States Department of

Energy under Contract DE-AC04-94AL85000.

Approved for public release, further dissemination unlimited. 
Issued by Sandia National Laboratories, operated for the United States Department of Energy by Sandia Corporation.

NOTICE: This report was prepared as an account of work sponsored by an agency of the United States Government. Neither the United States Government, nor any agency thereof, nor any of their employees, nor any of their contractors, subcontractors, or their employees, make any warranty, express or implied, or assume any legal liability or responsibility for the accuracy, completeness, or usefulness of any information, apparatus, product, or process disclosed, or represent that its use would not infringe privately owned rights. Reference herein to any specific commercial product, process, or service by trade name, trademark, manufacturer, or otherwise, does not necessarily constitute or imply its endorsement, recommendation, or favoring by the United States Government, any agency thereof, or any of their contractors or subcontractors. The views and opinions expressed herein do not necessarily state or reflect those of the United States Government, any agency thereof, or any of their contractors.

Printed in the United States of America. This report has been reproduced directly from the best available copy.

Available to DOE and DOE contractors from

Office of Scientific and Technical Information

P.O. Box 62

Oak Ridge, TN 37831

Prices available from (703) 605-6000

Web site: http://www.ntis.gov/ordering.htm

Available to the public from

National Technical Information Service

U.S. Department of Commerce

5285 Port Royal Rd

Springfield, VA 22161

NTIS price codes

Printed copy: A02

Microfiche copy: A01

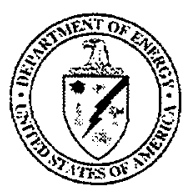




\section{DISCLAIMER}

Portions of this document may be illegible in electronic image products. Images are produced from the best available original document. 
SAND99-2692

Unlimited Release

Printed October 1999

FAST SOLUTIONS

OF

MAXWELL'S EQUATIONS

FOR

HIGH RESOLUTION ELECTROMAGNETIC IMAGING

OF

TRANSPORT PATHWAYS

\author{
Gregory A. Newman \\ Geophysical Technology Department \\ David M. Day \\ Applied Mathematics Department \\ Sandia National Laboratories \\ P.O. Box 5800 \\ Albuquerque, New Mexico 87185-0750
}

\begin{abstract}
A fast precondition technique has been developed which accelerates the finite difference solutions of the 3D Maxwell's equations for geophysical modeling. The technique splits the electric field into its curl free and divergence free projections, and allows for the construction of an inverse operator. Test examples show an order of magnitude speed up compared with a simple Jacobi preconditioner. Using this preconditioner a low frequency Neumann series expansion is developed and used to compute responses at multiple frequencies very efficiently. Simulations requiring responses at multiple frequencies, show that the Neumann series is faster than the than the preconditioned solution, which must compute solutions at each discrete frequency. A Neumann series expansion has also been developed in the high frequency limit along with spectral Lanczos methods in both the high and low frequency cases for simulating multiple frequency responses with maximum efficiency. The research described in this report was to have been carried out over a two-year period. Because of communication difficulties, the project was funded for first year only. Thus the contents of this report are incomplete with respect to the original project objectives.
\end{abstract}




\section{INTRODUCTION}

Each year, DOE invests on the order of $\$ 6$ billion to address the consequences of its past security activities. Not surprisingly, the ability to predict the transport of wastes and associated contaminants within the Earth's shallow crust has become of the most important challenges facing the Department. It is also one of the most daunting. Reliable prediction of the disposition of contaminants is critical for decisions on virtually every waste disposal option, from remediation technologies such as in-situ bioremediation to evaluations of the safety of nuclear waste repositories. Nuclear waste repository designs, both for highlevel and low-level wastes, require knowledge of transport of fluids, which is poorly understood, in both the vadose and saturated zones. Such predictive capabilities will impact the responsible utilization of our Nation's energy resources, particularly with respect to oil and gas. These predictions must be based on a comprehensive understanding of processes in the Earth's shallow crust. The task requires accurate predictions of the transport, migration, concentration, and disposition of contaminants. The computation of the transport and disposition, over long distances and over long times, of fluid-borne contaminants in the complex natural system is well beyond current capabilities. We currently lack the scientific understanding and computational algorithms necessary to address these issues with desired accuracy. Nevertheless, the newest generation of computational resources available at this laboratory provides an opportunity to address the scientific questions that are fundamental to high-resolution imaging of subsurface fluid transport and which ones are essential for improving our predictive capabilities.

The characterization and monitoring of fluid transport in terrestrial systems is difficult and expensive. The state-of-the-practice approach for direct characterization is based on borehole core and fluid sampling, and borehole logging. In addition to significant cost issues, analysis of borehole data raises significant questions, including: (1) The sampling basis is inherently very limited, and since the hydrology is very often complex with significant lateral and vertical variation, even major features that effect fluid flow and contaminant migration may not be delineated. (2) Each well potentially disrupts the subsurface opening new pathways for further transport flow and mobilization of contaminants by piercing impermeable layers. In both respects, indirect geophysical inverse or imaging methods offer advantages: they can potentially provide more complete information about fluid flow and contaminant transport and they are non-invasive. Here we focus on electromagnetic methods to image subsurface electrical conductivity because of their sensitivity to subsurface flow properties. While these methods cannot detect contaminants on the parts-per-million level, they have the potential to map migration pathways on the meter length scale. Unfortunately, conductivity imaging is a non-trivial problem; it is large scale (millions of parameters and thousands of data points), non-linear and requires significant computational resources, including both memory and time. Therefore, numerous approximations have been invoked in order to solve the imaging problem in a reasonable amount of time. Frequently used approximations such as migration methods, quasi-linear approximations, and asymptotic techniques limit the scope of the method and potentially introduce additional errors. Computational considerations also limit the ability to carry out an exhaustive analysis of the data, which is necessary to achieve maximum resolution and often it is not possible to perform more than a single inversion run or imaging experiment. In this case, no possibilities for alternative solutions can be tested, nor can the uncertainties of the model solution be quantified. To do so would require a large number of inversions. It is only recently that prototype solutions to the full problem have become available and it is now possible to image simple flow paths in well-characterized settings by using massively parallel (MP) computing capabilities at this laboratory. MP platforms, which provide for thousands of processors to operate on the problem simultaneously, have dramatically increased the rate at which the simulations can proceed and permit for the first time realistic imaging of 3D earth structure and transport pathways.

While the MP imaging capability discussed above overcomes the limitations of approximate imaging methods, much work remains in improving its resolution and efficiency. Currently images are produced at or near $10 . \mathrm{m}$ scale lengths that are ten times too coarse for detailed mapping of transport pathways. In terms of efficiencies it is still not viable to use this capability on a data set in an exhaustive manner. To do so is still prohibitive. The key point here is that the efficiency problem is closely related to resolution problem. Improvements in efficiency mean that the imaging code must employ finer meshes. It also allows one to excise it more frequently on data sets to ascertain the correct data weighting, fine tune stabilization parameters, allow for the possibilities to test alternative solutions and to determine the quality 
and quantity of data necessary for optimal results and resolution enhancement. To complete these tasks will require a corresponding effort be expended in developing the computationally efficient numerical procedures to conduct sophisticated three-dimensional imaging. It would include the study and implementation of rapid methods for solving complex systems of partial differential equations and techniques to accelerate the convergence rates of large-scale non-linear imaging schemes.

Solving the 3D non-linear inverse problem requires hundreds to thousands of forward modeling applications, which are necessary to compute predicted data, model sensitivities and function gradients. Moreover, the desire to image transport pathways at the meter length scale will require that fine meshes be employed and this will cause a corresponding increase in the computational demands needed to solve the forward problem. Thus, it can be seen that an inefficient solution to the forward problem will result in a computational bottleneck and initial efforts should be directed in reducing the time required for its solution. Our past experience has shown that application of finite difference methods to Maxwell's equations, are effective in simulating electromagnetic responses arising from complex 3D geology, where the sparse linear system of equations that arise are readily solved using iterative Krylov subspace methods. While these techniques work well for a modest number of forward modeling applications or for a single inversion nu, the computational times can be prohibitive if thousands of forward modeling applications or multiple inversion nuns are needed. Our solution to this problem is therefore to develop techniques to accelerate convergence rate of the Krylov subspace methods. In addition to exploring preconditioning for accelerating solutions at a discrete frequency, series-solution techniques and Lanczos methods will be investigated as a means of simulating solutions for multiple frequencies very efficiently.

\section{FORMULATION OF THE 3D ELECTROMAGNETIC FORWARD PROBLEM}

Assuming a time harmonic dependence of $e^{i \omega t}$, where $\omega$ is the angular frequency, a vector equation for the electric field for isotropic media, derived from Maxwell's equations in Newman and Alumbaugh (1995) is written as

$$
\nabla \times \nabla \times \mathbf{E}+i \omega \mu_{0}(\sigma+i \omega \varepsilon) \mathbf{E}=-i \omega \mu_{0} \mathbf{J}
$$

In equation (1), the electrical conductivity, dielectric permittivity and free space magnetic permeability are denoted by the symbols $\sigma, \varepsilon$ and $\mu_{0}$. Specification of the source vector $\mathbf{J}$ will depend if a total or scattered electric field solution is desired. In a scattered field formulation, we would set $\mathbf{E}=\mathbf{E}^{\mathbf{s}}$ in equation (1), and following Newman and Alumbaugh (1995) set

$$
\mathbf{J}=\left\{\left(\sigma-\sigma_{\mathrm{o}}\right)+i \omega\left(\varepsilon-\varepsilon_{\mathrm{o}}\right)\right\} \mathrm{E}^{\mathrm{b}}
$$

where $\sigma_{0}$ and $\varepsilon_{0}$ define the background conductivity and permittivity of uniform media and $\mathrm{E}^{\mathrm{b}}$ is the background electric field. Scattered field solutions are useful because in some applications they demonstrate improved accuracy over total field solutions (cf. Avdeev et al., 1999). We shall see that the price paid for a scattered field solution is an increase in the computational complexity needed to solve equation (1).

When equation (1) is approximated with finite differences using a Yee (1966) staggered grid, symmetrically scaled and Dirichlet boundary conditions applied (cf. Newman and Alumbaugh, 1995; Alumbaugh et al., 1996), a linear system results where,

$$
\mathbf{K} \mathbf{E}=\mathbf{S}
$$

The matrix $K$ is complex-symmetric and sparse with 13 non-zero entries per row and depends implicitly

on frequency. $\mathbf{S}$ is a discrete scaled representation of $-i \omega \mu_{0} \mathrm{~J}$. This system can be efficiently solved using the quasi minimum residual (qmr) method with simple Jocobi preconditioning. The qmr method belongs to the class of Krylov sub-space techniques that are highly efficient in iteratively solving sparse linear systems 
to a predetermined error level. The reader is referred to Newman and Alumbaugh, (1995) and Alumbaugh et al., (1996) for the details on how these solvers are implemented. Once the electric field is determined, the magnetic field is determined from Faraday's law by numerically approximating the curl of the electric field at the various nodal points and interpolating either the electric and magnetic nodal values to the point of interest. Finally, if the solution of equation (3) produces scattered fields, then background electric and magnetic fields must be added to the interpolated fields to yield the total fields.

\section{Development of a low frequency preconditioner}

It is well known that difficulties will be encountered when attempts are made to solve equation (1) as frequencies approach the static limit (cf. Alumbaugh et al., 1996; Smith 1996). Similar difficulties have been reported by Druskin et al. (1999) when equation (1) is solved with the spectral Lanczos decomposition method (SLDM), a method which also employs finite difference approximations. In this section we will show how these difficulties can be overcome with preconditioning. The preconditioner that we will introduce parallels the work of Druskin et al. (1999), who developed a new SLDM method with Krylov subspaces generated from the inverse of the Maxwell operator.

First we assume that we can neglect displacement currents. For most geological media, electromagnetic fields are not sensitive to the dielectric permittivity at frequencies below $10 \mathrm{MHz}$ (Hohmann and Ward, 1988). At these higher frequencies, solutions of the fields are obtained using the techniques outlined above or the new high frequency techniques discussed below. The steps necessary to design the low frequency preconditioner are as follows:

- divergence and curl free projections of the electric fields. Following LaBrecque (1995) and Druskin et al. (1999) we can then assume that the electric field can be decomposed into curl free and divergence free projections using the Helmholtz theorem, where

and

$$
\mathbf{E}=\Psi+\nabla \varphi
$$

$$
\nabla \cdot \Psi=0
$$

Upon substitution into equation (1) we find that

$$
-\nabla^{2} \Psi+i \omega \mu_{0} \sigma(\Psi+\nabla \varphi)=-i \omega \mu_{0} J
$$

where we used the vector identity

$$
\nabla \times \nabla \times \Psi=-\nabla^{2} \Psi
$$

since $\nabla \cdot \Psi=0$ and $\nabla \times \nabla \times \nabla \varphi=0$. The key idea behind this splitting of the electric field into curl free and divergence free projections is to deflate the null space of the curl-curl operator. When Krylov methods are applied directly to equation (3), this null space is responsible for the poor convergence as frequency approaches the static limit. It is also responsible for the "spurious mode" problem, where the gradient of a scalar potential can be added to the electric field and still satisfy the discrete version of equation (30) when the frequency is sufficiently small.

- approximate solutions at low induction numbers. To develop an approximate finite difference solution to equation (1) at low frequencies, we take the Euclidean norm of equation (1) where

$$
\left\|1 / \Delta^{2}+\omega \mu_{0} \sigma\right\|^{2}\|\mathbf{E}\|^{2} \geq\left\|\nabla \times \nabla \times \mathbf{E}+i \omega \mu_{0} \sigma \mathbf{E}\right\|^{2},
$$

where $\Delta$ is the characteristic grid size employed in the finite difference mesh. Thus the following inequality is deduced as frequency falls and the grid size is reduced,

$$
1 \gg \Delta^{2} \omega \mu_{0} \sigma_{\max },
$$


where $\sigma_{\max }$ is the maximum conductivity in the mesh. When the finite difference grid is non-uniform, $\Delta$ should be replaced by $\Delta_{\max }$, the maximum grid size used to approximate equation (1). Note that the right hand side of equation (8) is a dimensionless number and its square root is an induction number, which is an invariant parameter for diffusive electromagnetic fields. When frequency falls we increase the scale length and/or conductivity accordingly to have the fields remain invariant. Thus it appears that the induction number is more important in determining when equation (9) holds more so than frequency alone. It is also important to note than even at moderate frequencies, equation (9) may still hold if the grid size needed for the problem is sufficiently small. We have seen that this has direct implications for induction logging problems, which employ small grid sizes (cf. Avdeev et al., 1999). Now if equation (9) is satisfied, we can decouple equation (6), such that

$$
-\nabla^{2} \Psi=-i \omega \mu_{0} \mathbf{J}
$$

The boundary conditions required to solve equation (10) are a mixture of Dirichlet and Neumann types. Dirichlet conditions are applied to the tangential components of $\Psi$ on the mesh boundaries $\left(\Psi_{\mathfrak{l}}=0\right)$ and Neumann conditions on the normal components $\left(\partial \Psi_{n} / \partial n=0\right)$. These conditions enforce the requirement that $\nabla \cdot \Psi=0$ on the mesh boundaries and in turn within the solution domain since the divergence free field is required to satisfy the constraint equation

$$
\nabla^{2}(\nabla \cdot \Psi)=0
$$

Equation (11) follows by applying the divergence operator to equation (7). It is well known that when a function satisfies $u$ Laplace's equation, $\nabla^{2} u=0$, on some domain $\Omega$, with homogeneous boundary conditions of $u=0$ or $\partial u / \partial \mathrm{n}$ prescribed along the boundary $\Gamma$, it is identically zero on that domain. Note when applying the Neumann boundary condition, $n$, would specify the direction of the outward normal at the boundary. Because $\nabla \cdot \Psi=0$ on the boundary, we conclude for equation (11) that $\nabla \cdot \Psi=0$, everywhere.

The vector field $\Psi$ is not a complete solution to Maxwell's equations since it does not satisfy the auxiliary divergence condition on the current density with in the earth. To derive this condition we take the divergence of equation (1) and substitute in equation (4) to arrive at

$$
\nabla \cdot \sigma(\nabla \varphi)=-\nabla \cdot \sigma(\Psi)-\nabla \cdot \mathbf{J}
$$

Dirichlet boundary conditions will be applied to the discrete version of equation (12), where $\varphi=0$ on the mesh boundaries. When the air-earth interface is present, however, we employ the Neumann condition, $\partial \varphi / \partial n=0$, where $n$ specifies the direction of the outward normal at that interface. This later boundary condition enforces the constraint that current cannot leak from the earth into the air.

Approximate solutions of equation (1) at very small induction numbers can be obtained by first solving equation (10) followed by equation (12). Solution to these equations can be efficiently obtained using staggered finite difference methods with conjugate gradient methods to iteratively solve the systems to predetermined error levels.

- Solution preconditioning at small induction numbers. If it turns out that we cannot obtain a good approximate solution to equation (3) with a reasonable number of Krylov subspace iterations, or if such an approximate solution cannot be easily computed, we consider modifying the original problem to obtain a faster solution. This is the idea behind preconditioning, where we specify a preconditioning matrix, $\mathbf{M}$, and effectively solve the modified problem

$$
\mathbf{M}^{-1} \mathbf{K}=\mathbf{M}^{-1} \mathbf{S}
$$


At each step of the preconditioned algorithm, it is necessary to compute the product of $\mathbf{M}^{-1}$ with a vector, or, equivalently, to solve a linear system with the coefficient matrix $\mathbf{M}$, so $\mathbf{M}$ should be chosen so that such linear systems are much easier to solve than the original problem. Moreover, the properties we desire in a preconditioner for non-Hermitian matrix iterations, including qmr and other related methods, are that the preconditioned matrix should somehow approximate the identity (Greenbaum, 1997). It is therefore obvious that the approximate scheme discussed above could be very effective in preconditioning equation (3) at moderate to low induction numbers. If this idea is to be practical, however, it will be necessary to find fast methods to solve the approximate equations. A good preconditioner is not simply based on a relatively low dimensional Krylov subspace, but also on the time required to construct it. Implementation of the preconditioner is straight forward. We first convert equations (10) and (12) into discrete matrix equations via staggered finite differences. In the preconditioned qmr algorithm, at each iteration, we then substitute the residual, defined by $\mathbf{r}=\mathbf{K} \mathbf{E}-\mathbf{S}$ into the right hand side of the discrete version of equation (10). Equation (10) is then solved followed by equation (12). Fast preconditioned conjugate gradient techniques employing incomplete Cholesky factorization have proven quite effective in solving these equations. Furthermore we have determined that it is not necessary to precisely solve these equations. Test examples show that we only need to approximately solve these equations for a significant impact on reducing the time required for solving equation (3).

- Preconditioner effectiveness. Equation (9) provides only a rough measure on the effectiveness of the preconditioner. We need to estimate the largest and smallest non-zero values that the discrete curl-curl operator can assume. Estimating the largest and smallest non-zero eigenvalues of this operator is therefore necessary to determine when the preconditioner will be the most and least effective. Consider equation (3), when $\omega=0$. Using the maximum row sum, we find the largest eigenvalue of the discrete curl-curl operator satisfies

$$
\lambda_{\max } \approx 13 / \Delta_{\min }^{2} \text {, }
$$

where $\Delta_{\min }$ is the minimum grid size used in the mesh. The corresponding minimum eigenvalue is estimated in the limit as the grid size $\Delta \rightarrow 0$, as

$$
\lambda_{\min } \approx 2 \pi^{2} \Lambda_{\max }^{2},
$$

with $\mathrm{L}_{\max }$ as the largest dimension of the 3D Cartesian mesh. Given the largest eigenvalue, the inequality in equation (9) is written as

$$
1>\omega \mu_{0} \sigma_{\max } \Delta_{\min }^{2} / 13 \text {, }
$$

which provides the optimistic measure when the preconditioner will be most effective. In the worst case, which corresponds to the smallest non-zero eigenvalue, we have, however,

$$
1 \gg \omega \mu_{0} \sigma_{\max } L_{\max }^{2} / 2 \pi^{2}
$$

Unfortunately, equation (17) shows that reducing the grid size $\Delta$ does not provide any additional benefit in accelerating the solution of equation (3).

\section{A low frequency series solution} expression

The preconditioner just introduced is the first term of a Neumann series expansion applied to the

$$
\mathbf{E}=\left(\mathbf{I}+\omega \mathbf{A}^{+} \mathbf{D}\right)^{-1} \mathbf{A}^{+} \mathbf{S}
$$


Here $\mathbf{A}^{+}$represents the inverse of the discrete curl-curl operator after we have deflated its null space, where

$$
\mathbf{K}=\mathbf{A}+\omega \mathbf{D},
$$

where $\mathbf{A}$ and $\mathbf{D}$ are matrices that represent the discrete curl-curl operator and attenuation terms in equation (1). The matrix $\mathbf{D}$ is also diagonal with imaginary elements. Note that the matrix-vector product $\mathrm{A}^{+} \mathrm{S}$ is determined by solving discrete versions of equations (10) and (12) and then combining these solutions according to equation (4), where $S$ represents the discrete right hand side of equation (10). The Neumann series expansion of equation (18) can then be written as

$$
\mathbf{E}=\sum_{\mathbf{n}=0}^{\infty}\left\{-\omega \mathbf{A}^{+} \mathbf{D}\right\}^{\mathbf{n}} \mathbf{A}^{+} \mathbf{S}
$$

This series will converge provided the spectral radius

$$
\rho\left(\omega \mathbf{A}^{+} \mathbf{D}\right)<1 .
$$

Note that if the conductivity of the medium, $\sigma$, is constant we can estimate the spectral radius as

$$
\rho\left(\omega \mathbf{A}^{+} \mathbf{D}\right) \approx \omega \mu_{0} \sigma \mathrm{L}_{\max }^{2} / 2 \pi^{2},
$$

which is identical to expression provided in equation (17) for studying the behavior of the low frequency preconditioner, if $\sigma=\sigma_{\max }$. Provided the aforementioned series converges, it permits an efficient way to estimate multiple frequency responses, since

$$
\mathbf{E}=\mathbf{A}^{+} \mathbf{S}-\omega\left\{\mathbf{A}^{+} \mathrm{D}\right\} \mathbf{A}^{+} \mathbf{S}+\omega^{2}\left\{\mathbf{A}^{+} \mathrm{D}\right\}\left\{\mathbf{A}^{+} \mathrm{D}\right\} \mathbf{A}^{+} \mathbf{S}-
$$

or

$$
\mathbf{E}=\varepsilon_{0}-\omega \varepsilon_{1}+\omega^{2} \varepsilon_{2}-\omega^{3} \varepsilon_{3}+\ldots \ldots
$$

where

$$
\begin{aligned}
\boldsymbol{\varepsilon}_{0}=\mathbf{A}^{+} \mathbf{S} \\
\boldsymbol{\varepsilon}_{1}=\mathbf{A}^{+} \mathbf{D} \varepsilon_{0} \\
\boldsymbol{\varepsilon}_{2}=\mathbf{A}^{+} \mathbf{D} \varepsilon_{1} \\
\boldsymbol{\varepsilon}_{3}=\mathbf{A}^{+} \mathbf{D} \varepsilon_{2} \\
\quad \cdot \\
\quad \\
\boldsymbol{\varepsilon}_{\mathrm{it1}}=\mathbf{A}^{+} \mathbf{D} \boldsymbol{\varepsilon}_{\mathrm{i}} .
\end{aligned}
$$

In the above expressions it is assumed that $\mathbf{S}$ displays a simple dependence on $\omega$ such that 


$$
\mathbf{S}(\omega)=\omega \mathbf{S}_{\mathbf{o}},
$$

which is typical for total field problems, where $J$ in equation (1) does not depend upon frequency. With scattered field solutions this is not the case and it is necessary to first expand $\mathbf{S}$ in a convergent power series in $\omega$, where

$$
\mathbf{S}=\mathbf{S}_{\mathbf{0}}+\omega \mathbf{S}_{1}+\omega^{2} \mathbf{S}_{2}+\omega^{3} \mathbf{S}_{3}+\ldots \ldots
$$

By substituting equation (27) into equation (20) and equating powers in $\omega$, we find

$$
\begin{gathered}
\varepsilon_{o}=A^{+} S_{o} \\
\varepsilon_{1}=A^{+}\left\{D \varepsilon_{o}-S_{1}\right\} \\
\varepsilon_{2}=A^{+}\left\{D \varepsilon_{1}+S_{2}\right\} \\
\varepsilon_{3}=A^{+}\left\{D \varepsilon_{2}-S_{3}\right\} \\
: \\
\cdot \\
\varepsilon_{i+1}=A^{+}\left\{D \varepsilon_{i} \pm S_{i+1}\right\}
\end{gathered}
$$

where the electric field is still determined from equation (24). Note that in equation (28) when $i$ is even then $\varepsilon_{i+1}=A^{+}\left\{D \varepsilon_{i}-S_{i+1}\right\}$ and when $i$ is odd $\varepsilon_{i+1}=A^{+}\left\{D \varepsilon_{i}+S_{i+1}\right\}$.

\section{A high frequency series solution}

Let us now write the solution to equation (3) in terms of a Neumann series in the high frequency limit; $\omega \rightarrow \infty$. Since we are still neglecting displacement currents we use equation (19) to recast equation (3) as

$$
\mathbf{E}=1 / \omega\left(\mathbf{I}+1 / \omega \mathbf{D}^{+} \mathbf{A}\right)^{-1} \mathbf{D}^{+} \mathbf{S},
$$

where we formally write the inverse of the matrix $\mathbf{D}$ as $\mathbf{D}^{+}$since it is singular in perfect insulators, such as the air, because the conductivity is zero there. For the moment let us assume that the air or other perfect insulators are not present in the model so we can write $\mathbf{D}^{+}=\mathbf{D}^{-1}$ hence $\mathbf{D}^{+}$is no longer singular. Thus

$$
\mathbf{E}=1 / \omega \sum_{\mathbf{n}=0}^{\infty}\left\{-1 / \omega \mathbf{D}^{+} \mathbf{A}\right\}^{\mathrm{n}} \mathbf{D}^{+} \mathbf{S}
$$

This series will converge if the spectral radius

$$
\rho\left(\mathbf{D}^{+} \mathbf{A} / \omega\right)<1 .
$$

Note that if the conductivity of the medium, $\sigma$, is constant and we assume that the smallest grid size employed in the mesh is $\Delta_{\min }$ then we have

$$
\rho\left(\mathrm{D}^{+} \mathbf{A} / \omega\right) \approx 13 /\left(\omega \mu_{0} \sigma \Delta_{\min }{ }^{2}\right) .
$$


Given that the high frequency series converges, we can again obtain multiple frequency responses very efficiently since

$$
\mathbf{E}=\omega^{-1} \mathbf{D}^{+} \mathbf{S}-\omega^{-2}\left\{\mathbf{D}^{+} \mathbf{A}\right\} \mathbf{D}^{+} \mathbf{S}+\omega^{-3}\left\{\mathbf{D}^{+} \mathbf{A}\right\}\left\{\mathbf{D}^{+} \mathbf{A}\right\} \mathbf{D}^{+} \mathbf{S}-\ldots \ldots \ldots
$$

where

$$
\mathbf{E}=\omega^{-1} \boldsymbol{\varepsilon}_{0}-\omega^{-2} \varepsilon_{1}+\omega^{-3} \varepsilon_{2}-\omega^{-1} \varepsilon_{3}+\ldots \ldots
$$

where

$$
\begin{aligned}
\varepsilon_{1}= & \mathbf{D}^{+} \mathbf{S} \\
\varepsilon_{2}= & \mathbf{D}^{+} \mathbf{A} \varepsilon_{1} \\
\varepsilon_{3}= & \mathbf{D}^{+} \mathbf{A} \varepsilon_{2} \\
\varepsilon_{4}= & \mathbf{D}^{+} \mathbf{A} \varepsilon_{3} \\
& \cdot \\
& \cdot \\
& \cdot \\
\varepsilon_{\mathrm{i}+1} & =\mathbf{D}^{+} \mathbf{A} \varepsilon_{i} .
\end{aligned}
$$

In the above expressions it is again assumed that $\mathbf{S}$ displays a simple dependence on $\omega$ such that

$$
\mathbf{S}(\omega)=\omega \mathbf{S}_{\mathbf{0}}
$$

With scattered field solutions this is not the case and it is necessary to first expand $\mathbf{S}$ in a convergent power series in $\omega^{-1}$ and substitute the resulting series into equation (30) and equate terms in powers of $\omega^{-1}$. The reader will recall that a similar procedure was also used in the low frequency series expansion.

The high frequency series cannot be used when air is present as a material property in the matrix D. To obtain a high frequency series when air is present we first split the electric field between that of the air and that of the earth using the Schur complement approach. Hence equation (3) is now expressed as

$$
\left(\begin{array}{cc}
\mathbf{A}_{a} & \mathbf{K}_{a e} \\
\mathbf{K}_{e a} & \mathbf{A}_{e}+\omega \mathbf{D}_{e}
\end{array}\right)\left(\begin{array}{l}
\mathbf{E}_{a i r} \\
\mathbf{E}_{e a r t h}
\end{array}\right)=\left(\begin{array}{l}
\mathbf{S}_{a i r} \\
\mathbf{S}_{e a r h}
\end{array}\right)
$$

where the matrices in equation (37) include the discrete curl-curl operator in the air and the earth, $\mathbf{A}_{a}$ and $A_{e}$, the attenuation matrix $D_{e}$ within the earth, which is not singular, and matrices $K_{e a}$ and $K_{\alpha e}$ which couple the fields at the air-earth interface. These coupling matrices are highly sparse. Note also that 


$$
\mathbf{K}_{a e}=\mathbf{K}_{e a}^{\mathbf{T}}
$$

since the matrix $K$ is symmetric in equation (3). Thus we can now eliminate the fields in the air and solve for only the fields within the earth, where

$$
\left\{A_{c}+\omega D_{e}-K_{e a} A_{a}^{+} K_{a e}\right\} E_{\text {earth }}=S_{\text {aeatb }}-K_{e a} A_{a}^{+} S_{\text {air }}
$$

Here $\mathbf{A}_{a}{ }^{+}$represents the inverse of the discrete curl-curl operator in the air after we have deflated its null space. Note also that the matrix $\left\{\mathbf{A}_{e}+\omega \mathbf{D} e-\mathbf{K}_{e a} \mathbf{A}_{a}{ }^{+} \mathbf{K}_{\boldsymbol{}}\right\}$ is similar to the matrix $\mathbf{K}$ in equation (3) as both matrices are complex symmetric. The electric field within the earth is now expressed as

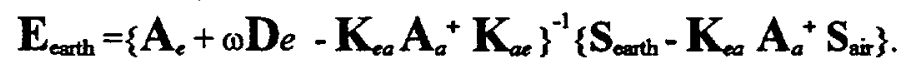

Equation (40) can also be expanded in a formal Neumann series. To see this we recast it such that

$$
\mathbf{E}_{\text {earth }}=\left\{\mathbf{I}+1 / \omega \mathbf{D} e^{-1}\left(\mathbf{A}_{e}-\mathbf{K}_{e a} \mathbf{A}_{a}^{+} \mathbf{K}_{a e}\right)\right\}^{-1} \mathbf{D}^{-1}\left\{\mathbf{S}_{\text {earth }}-\mathbf{K}_{e a} \mathbf{A}_{a}^{+} \mathbf{S}_{\mathrm{ari}}\right\} / \omega
$$

Hence

$$
\mathbf{E}_{\text {earth }}=1 / \omega \sum_{\mathbf{n}=0}^{\infty}\left\{-1 / \omega \mathbf{D}^{-1}\left(\mathbf{A}_{c}-\mathbf{K}_{e a} \mathbf{A}_{a}^{+} \mathbf{K}_{a c}\right)\right\}^{\mathrm{n}} \mathbf{D}^{-1}\left(\mathbf{S}_{\text {earth }}-\mathbf{K}_{c a} \mathbf{A}_{a}^{+} \mathbf{S}_{\text {axi }}\right)
$$

The above series will converge provided that the spectral radius

$$
\rho\left(\mathbf{D}^{-1}\left(\mathbf{A}_{e}-\mathbf{K}_{e \mathrm{c}} \mathbf{A}_{a}^{+} \mathbf{K}_{a c}\right) / \omega\right)<1 .
$$

Provided the aforementioned series converges, it permits an efficient means to compute multiple frequency responses because for a total field solution we would have

$$
\begin{aligned}
& \mathbf{E}_{\text {earth }}=1 / \omega \mathbf{D}^{-3}\left(\mathbf{S}_{\text {earth }}-\mathbf{K}_{c a} \mathbf{A}_{\sigma}^{+} \mathbf{S}_{\text {air }}\right) \\
& -1 / \omega^{2}\left\{\mathbf{D}^{-1}\left(\mathbf{A}_{e}-\mathbf{K}_{c a} \mathbf{A}_{a}^{+} \mathbf{K}_{a c}\right)\right\} \mathbf{D}^{-1}\left(\mathbf{S}_{\text {earth }}-\mathbf{K}_{e a} \mathbf{A}_{a}^{+} \mathbf{S}_{\mathrm{axi}}\right) \\
& +1 / \omega^{3}\left\{\mathbf{D}^{-1}\left(\mathbf{A}_{e}-\mathbf{K}_{e a} \mathbf{A}_{a}^{+} \mathbf{K}_{c e}\right)\right\}\left\{\mathbf{D}^{-1}\left(\mathbf{A}_{e}-\mathbf{K}_{e a} \mathbf{A}_{a}^{+} \mathbf{K}_{a e}\right)\right\} \mathbf{D}^{-1}\left(\mathbf{S}_{\text {earth }}-\mathbf{K}_{e a} \mathbf{A}_{a}^{+} \mathbf{S}_{\text {ain }}\right) \\
& -\ldots . . .
\end{aligned}
$$

when ( $\mathbf{S}_{\text {earth }}-\mathbf{K}_{e a} \mathbf{A}_{a}^{+} \mathbf{S}_{\mathrm{arr}}$ ) displays first order dependence on $\omega$, as in equation (26). Once again to obtain a scattered field series solution it will be necessary to first expand $\left(S_{\text {earth }}-K_{e a} A_{a}^{+} S_{\text {air }}\right)$ in a power series in $\omega^{-1}$ and substitute the resulting series into equation (42) and equate terms in powers of $\omega^{-1}$.

\section{Spectral Lanczos Solutions}

We recall that we are formally solving the following linear systems

$$
\dot{E}=\left(\mathbf{I}+\omega \mathbf{A}^{+} \mathbf{D}\right)^{-1} \mathbf{A}^{+} \mathbf{S},
$$

in the low frequency case and

$$
\mathbf{E}=\left(\omega \mathbf{I}+\mathbf{D}^{+} \mathbf{A}\right)^{-1} \mathbf{D}^{+} \mathbf{S},
$$


in the high frequency case. When the air is present, however, we recast equation (46) as

$$
\mathbf{E}_{\text {earth }}=\left\{\omega \mathbf{I}+\mathbf{D}^{-1}\left(\mathbf{A}_{e}-\mathbf{K}_{e a} \mathbf{A}_{a}^{+} \mathbf{K}_{a e}\right)\right\}^{-1} \mathbf{D e}^{-1}\left\{\mathbf{S}_{\text {earth }}-\mathbf{K}_{e a} \mathbf{A}_{a}^{+} \mathbf{S}_{\text {air }}\right\}
$$

If the sources $\mathbf{A}^{+} \mathbf{S}, \mathbf{D}^{+} \mathbf{S}$ and $\mathbf{D}^{-1}\left\{\mathbf{S}_{\text {earth }}-\mathbf{K}_{e c} \mathbf{A}_{a}^{+} \mathbf{S}_{\mathrm{air}}\right\}$, display a simple dependence on $\omega$, as in equation (26), spectral Lanczos methods, using a reduced model approximation, can compute these solutions for multiple frequencies, with far more accuracy and efficiency than the Neumann series solutions outlined above (cf., Druskin et al. 1999).

In deriving the Lanczos algorithm, let us first consider the low frequency case (equation (45)) and its Neumann series expansion,

$$
\mathbf{E}=\sum_{n=0}^{m-1}\left\{-\omega \mathbf{A}^{+} \mathbf{D}\right\}^{n} \mathbf{A}^{+} \mathbf{S}+\sum_{n=m}^{\infty}\left\{-\omega \mathbf{A}^{+} \mathbf{D}\right\}^{n} \mathbf{A}^{+} \mathbf{S}
$$

Let us now describe the source $\mathrm{A}^{+} \mathrm{S}=\omega \mathrm{A}^{+} \mathrm{S}_{0}$ in the following expansion

$$
\omega \mathbf{A}^{+} \mathbf{S}_{\mathbf{0}}=\omega \beta_{1} \mathbf{Q}_{m} \mathbf{e}_{\mathbf{1}}
$$

where

$$
\mathbf{e}_{1}=\left(\begin{array}{l}
1 \\
0 \\
0 \\
\cdot \\
0
\end{array}\right)_{m \times 1}
$$

is a vector that selects the first of $m$ Lanczos vector, $\mathbf{q}_{1}$, which are collectively stored in the matrix $\mathbf{Q}_{m}$, where

$$
\mathbf{Q}_{m}=\left(\mathbf{q}_{1} \mathbf{q}_{2} \mathbf{q}_{3} \ldots \ldots \mathbf{q}_{m}\right)
$$

The constant, $\beta_{1}$, and the Lanczos vectors, which have dimension $n x 1$, where $n$ is the number of electric field unknowns are determined from the Lanczos process described below. After $m$ steps of the Lanczos process a tridiagonal matrix $T_{m}$ is also produced 


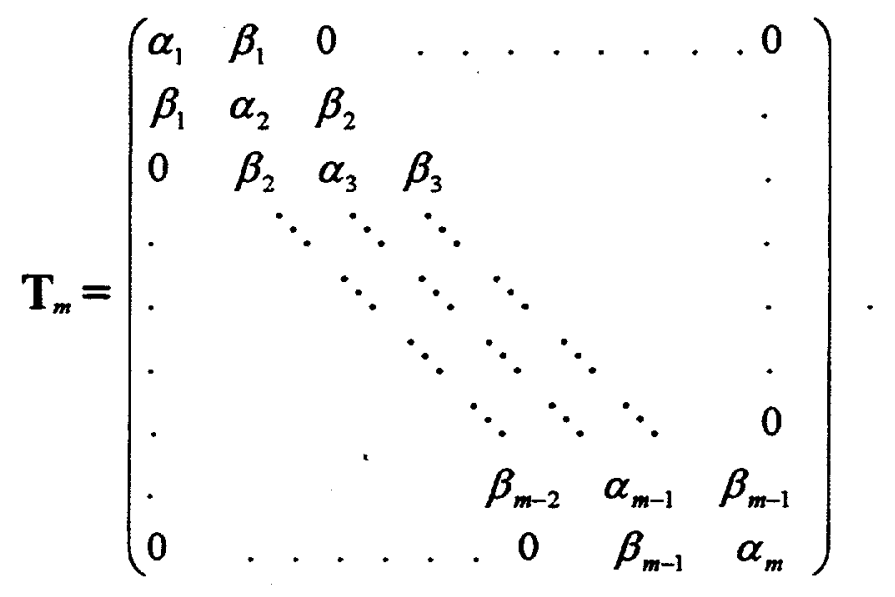

It is known as the number of Lanczos steps , $m$, increases, the eigenvalues of $\mathbf{T}_{m}$, which is dimension $m x m$, will converge to the eigenvalues of matrix $\mathbf{A}^{+} \mathbf{D}$ in exact arithmetic (Golub and Van Loan, 1989). Another pleasing property of the of the matrix $\mathbf{T}_{m}$ is the following relation, which is proved in Appendix,

$$
\left(\mathbf{A}^{+} \mathbf{D}\right)^{\mathrm{j}} \mathbf{A}^{+} \mathbf{S}_{\mathbf{0}}=\beta_{1} \mathbf{Q}_{m} \mathbf{T}_{m}^{\mathrm{j}} \mathbf{e}_{\mathbf{1}}, \quad \mathrm{j}=0,1,2, \ldots, m-1
$$

Thus when equation (53) is substituted into equation (48), using the fact that $A^{+} S=\omega A^{+} S_{0}$, we find

$$
\mathbf{E}=\omega \beta_{1} \mathbf{Q}_{m} \sum_{n=0}^{m-1}\left\{-\omega \mathbf{T}_{m}\right\}^{\mathrm{n}} \mathbf{e}_{1}+\sum_{n=m}^{\infty}\left\{-\omega \mathbf{A}^{+} \mathbf{D}\right\}^{\mathrm{n}} \mathbf{A}^{+} \mathbf{S}
$$

This equation can also be expressed as

$$
\mathbf{E}=\omega \beta_{1} \mathbf{Q}_{m} \sum_{n=0}^{\infty}\left\{-\omega \mathbf{T}_{m}\right\}^{n} \mathbf{e}_{1}+\sum_{n=m}^{\infty}\left\{-\omega \mathbf{A}^{+} \mathbf{D}\right\}^{n} \mathbf{A}^{+} \mathbf{S}-\omega \beta_{1} \mathbf{Q}_{m}\left\{-\omega \mathbf{T}_{m}\right\}^{n} \mathbf{e}_{\mathbf{1}}
$$

Assuming that a sufficient number of Lanczos steps are taken, we can ignore the second sum in the above equation and express the electric field using a reduced model approximation, as

$$
\mathbf{E} \approx \omega \beta_{1} \mathbf{Q}_{m}\left(\mathbf{I}_{m}+\omega \mathbf{T}_{m}\right)^{-1} \mathbf{e}_{1}
$$

where we desire that $m<n$. Because $m<<n$ it is much easier to solve equation (55) instead of equation (45). Moreover we can readily compute the eigenvalues and eigenvectors of $T_{m}$ and thereby diagonalize it,

$$
\mathbf{T}_{m}=\mathbf{U}_{m} \Lambda_{m} \mathbf{U}_{m}^{\mathbf{T}}
$$

where the eignevectors are orthonormal, $\mathbf{U}_{m}^{\mathbf{T}} \mathbf{U}_{m}=\mathbf{I}_{m}$, and the eignevalues are stored within the diagonal matrix $\Lambda_{m}$. Thus we can write equation (56) in a form from which multiple frequencies can be readily computed, very efficently, 


$$
\mathbf{E} \approx \omega \beta_{1} \mathbf{Q}_{m} \mathbf{U}_{m}\left(\mathbf{I}_{m}+\omega \Lambda_{m}\right)^{-1} \mathbf{U}_{m}^{\mathbf{T}} \mathbf{e}_{\mathbf{l}}
$$

To derive the Lanczos algorithm, we consider the following eigenvalue problem

$$
\mathbf{A}^{+} \mathbf{D} \mathbf{v}=\lambda \mathbf{v}
$$

and assume that $\mathbf{A}^{+}=\mathbf{L}^{-\mathrm{T}} \mathbf{L}^{-1}$, where $\mathbf{L}$ is a lower triangular matrix. Thus

$$
\mathbf{L}^{-\mathbf{T}} \mathbf{L}^{-1} \mathbf{D} \mathbf{v}=\lambda \mathbf{v}
$$

If we premultiply the above expression by $\mathbf{L}^{\mathbf{T}}$ we find that

$$
\mathbf{L}^{-1} \mathbf{D} \mathbf{v}=\lambda \mathbf{L}^{\mathbf{T}} \mathbf{v}
$$

and if we set $y=\mathbf{L}^{\mathbf{T}} \mathbf{v}$, we have

$$
\mathbf{L}^{-1} \mathbf{D} \mathbf{L}^{-\mathbf{T}} \mathbf{y}=\lambda \mathbf{y}
$$

Thus the symmetric matrix, $\mathbf{L}^{-1} \mathbf{D} \mathbf{L}^{-\mathrm{T}}$, has the same eigenvalues as the matrix $\mathbf{A}^{+} \mathbf{D}$ in equation (59) through the similarity transformation. We can therefore apply the symmetric Lanczos process to the matrix, $\mathbf{L}^{-1} \mathbf{D} \mathbf{L}^{-T}$, where we have assume that we have completed its tridiagonalization. Thus from Golub and Van Loan (1989), we have

$$
\mathbf{L}^{-1} \mathbf{D} \mathbf{L}^{-\mathbf{T}} \mathbf{V}=\mathbf{V} \mathbf{T}
$$

Here the $\mathbf{V}$ are Lanczos vectors derived from the Lanczos algorithm, applied to symmetric matrix $\mathbf{L}^{-1} \mathbf{D} \mathbf{L}^{-\mathbf{T}}$, where

$$
\mathbf{V}^{\mathbf{T}} \mathbf{V}=\mathbf{I}
$$

If we now set

$$
\mathbf{Q}=\mathbf{L}^{-\mathbf{T}} \mathbf{V}
$$

then equation (62) is recasted as

$$
\mathbf{L}^{-1} \mathbf{D} \mathbf{Q}=\mathbf{V} \mathbf{T}
$$

and it we now premultiply by $\mathbf{L}^{-\mathrm{T}}$ we find

$$
\mathbf{L}^{-\mathbf{T}} \mathbf{L}^{-1} \mathbf{D} \mathbf{Q}=\mathbf{Q} \mathbf{T}
$$

Hence

$$
\mathbf{A}^{+} \mathbf{D} \mathbf{Q}=\mathbf{Q} \mathbf{T}
$$




$$
\mathbf{D Q}=\mathbf{A Q T} \text {. }
$$

Finally when we premultiply equation (69) by $\mathbf{Q}^{\mathrm{T}}$ and use equation (65) we find that

$$
\mathbf{V}^{\mathrm{T}} \mathbf{L}^{-1} \mathbf{D}^{-\mathrm{T}} \mathbf{V}=\mathbf{Q}^{\mathrm{T}} \mathbf{A Q} \mathbf{T}
$$

from which we conclude that

$$
\mathbf{Q}^{\mathrm{T}} \mathbf{A Q}=\mathbf{I}
$$

on account of equations (63) and (64). The point of these derivations is that we can now derive a variant of the symmetric Lanczos algorithm that enforces equation (71) using equation (69).

To derive the alogrithm we equate columns of $\mathbf{D Q}=\mathbf{A Q T}$, where

$$
\mathbf{D q} \mathbf{q}_{\mathrm{i}}=\mathbf{A}\left\{\beta_{j} \mathbf{q}_{\mathrm{i}-1+\alpha_{j}} \mathbf{q}_{\mathrm{i}}+\beta_{j+1} \mathbf{q}_{j+1}\right\}
$$

and set

$$
\mathbf{r}_{\mathrm{j}}=\mathbf{D q} \mathbf{q}_{\mathrm{j}-\alpha_{\mathrm{j}}} \mathbf{A q _ { \mathrm { i } } - \beta _ { \mathrm { j } }} \mathbf{A q _ { \mathrm { j } - 1 }}
$$

where $r_{j}=\beta_{j+1} A q_{j+1}$. When we enforce the orthogonality condition in equation (70) we find that

$$
\alpha_{j}=\mathbf{q}_{j}{ }^{\mathbf{T}} \mathbf{D} \mathbf{q}_{j}
$$

When $\mathbf{r}_{\mathbf{j}}$ is known, we also find that $\mathbf{q}_{j+1}$ is determined from

$$
\mathbf{q}_{\mathrm{j}+1}=\mathbf{A}^{+} \mathbf{r}_{j} / \beta_{\mathrm{j}+1},
$$

where $\beta_{j}$ is determined form the condition $\mathbf{q}_{j+1}{ }^{\mathbf{T}} \mathbf{A} \mathbf{q}_{\mathrm{j}+1}=I$ and is found to be

$$
\beta_{\mathrm{j}+1}^{2}=\mathbf{r}_{\mathrm{j}}^{\mathbf{T}} \mathbf{A}^{+} \mathbf{r}_{\mathrm{j}} .
$$

Thus we arrive at the following $m$ step Lanczos algorithm

1) Set $q_{0}=0$ and i=1. Given $A^{+} S_{0}=\beta_{1} q_{1}$, set $\beta_{1}=\sqrt{S_{0}{ }^{T} A^{+} S_{0}}$ so that $q_{1}{ }^{T} A q_{1}=I$.

2) $\alpha_{i}=\mathbf{q}_{i}{ }^{\mathrm{T}} \mathbf{D} \mathbf{q}_{\mathrm{i}}$

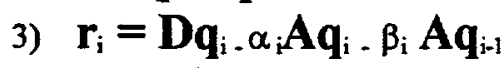

4) $\mathbf{s}_{\mathrm{i}}=\mathbf{A}^{+} \mathbf{r}_{\mathrm{i}}$

5) $\beta_{i+1}=\sqrt{\mathbf{r}_{i}^{\mathbf{T}_{s_{i}}}}$

6) $q_{j+1}=s_{i} / \beta_{j+1}$

7) if $\mathbf{i} \leq \mathrm{m}$ go to step 3 , otherwise stop 
When we consider equation (46) and use its high frequency series expansion, the reduced model. approximation after $m$ Lanczos steps can be written as

$$
\mathbf{E} \approx \omega \beta_{1} \mathbf{Q}_{m}\left(\omega \mathbf{I}_{m}+\mathbf{T}_{m}\right)^{-1} \mathbf{e}_{1}
$$

where

$$
D^{+} \mathbf{S}=\omega D^{+} S_{o}=\omega \beta_{1} Q_{m} \mathbf{e}_{1}
$$

The $m$ step Lanczos algorithm for the high frequency series expansion is given below.

1) Set $\mathbf{q}_{0}=\mathbf{0}$ and $\mathrm{i}=1$. Given $\mathbf{D}^{+} \mathbf{S}=\boldsymbol{\beta}_{1} \mathbf{q}_{1}$, set $\boldsymbol{\beta}_{1}=\sqrt{\mathbf{S}_{0}{ }^{\mathbf{T}} \mathbf{D}^{+} \mathbf{S}_{0}}$ so that $\mathbf{q}_{1}{ }^{\mathrm{T}} \mathbf{D} \mathbf{q}_{1}=\mathbf{I}$

2) $\alpha_{\mathrm{i}}=\mathbf{q}_{\mathrm{i}}^{\mathbf{T}} \mathbf{A} \mathbf{q}_{\mathrm{i}}$

3) $\mathbf{r}_{\mathrm{i}}=\mathbf{A} \mathbf{q}_{\mathrm{i}-\alpha_{\mathrm{i}}} \mathbf{D} \mathbf{q}_{\mathrm{i}-\beta_{\mathrm{i}}} \mathbf{D} \mathbf{q}_{\mathrm{i}-1}$

4) $\mathbf{s}_{\mathrm{i}}=\mathbf{D}^{+} \mathbf{r}_{\mathrm{i}}$

5) $\beta_{i+1}=\sqrt{\mathbf{r}_{\mathbf{i}} \mathbf{T}_{\mathbf{i}}}$

6) $\mathbf{q}_{\mathrm{i}+1}=\mathbf{s}_{\mathrm{i}} / \beta_{\mathrm{j}+1}$

7) if $\mathrm{i} \leq \mathrm{m}$ go to step 3 , otherwise stop

Finally when the air is present in the model, and we desire the reduced model approximation of equation (47), we would replace $A$ by $A_{e}-K_{e a} A_{a}^{+} K_{a e}$ and $S$ by $S_{e a r t h}-K_{e a} A_{a}^{+} S_{\text {air, }}$ where $D^{+}=D_{e}^{-1}$ in the above algorithm. Note that $n$ is now the number of electric field unknowns within the earth.

\section{SOME RESULTS}

We now demonstrate the effectiveness of the low frequency preconditioner derived above. The model we consider arises from an induction logging problem. The mesh employed consists of 134,832 grid points that sample 337,376 electric field unknowns on a domain that is $16 \times 6 \times 16 \mathrm{~m}$ in the $\mathrm{x}, \mathrm{y}$ and $\mathrm{z}$ coordinate directions. At a frequency of $160 \mathrm{kHz}$ the solution time needed for the finite difference (FD) scattered field solution to achieve an residual of $10^{-10}$, defined by $\|\mathbf{K E}-\mathbf{S}\|^{2} /\|\mathbf{S}\|^{2}$, was 1672 sec for an IBM RS-6000 590 workstation when simple Jacobi preconditioning was employed. When the problem is solved using the low frequency preconditioner, the solution time was $187 \mathrm{sec}$, nearly an order of magnitude faster. In Table 1, we study the effectiveness of the preconditioner over three decades of frequency. As expected, the preconditioner is most effective at the lower frequencies. For the highest frequency, $5 \mathrm{MHz}$, we observed that the preconditioner can actually fail to converge, and may also result in much slower solution times than a solution that employs simple Jacobi precnditioning. In this example, the upper bound given by equation (16) provides a good measure when to use the precondioner, provided the left hand side of equation (16) is at least two orders of magnitude smaller than its right hand side. The lower bound expression, on the other hand (equation (17), is too pessimistic. 
TABLE 1

\begin{tabular}{|c|c|c|c|c|c|}
\hline Frequency & Solution Time & qmr Iterations & Residual & Equation (16) & Equation (17) \\
\hline $10 \mathrm{kHz}$ & $148 \mathrm{sec}$ & 3 & $2 \mathrm{e}-09$ & $3.0 e-04$ & 20.5 \\
\hline $40 \mathrm{kHz}$ & $185 \mathrm{sec}$ & 4 & $5 e-11$ & $1.2 e-03$ & 82 \\
\hline $80 \mathrm{kHz}$ & $184 \mathrm{sec}$ & 4 & $1.5 e-10$ & $2.4 \mathrm{e}-03$ & 164 \\
\hline $160 \mathrm{kHz}$ & $187 \mathrm{sec}$ & 4 & $1.8 \mathrm{e}-10$ & $4.8 \mathrm{e}-03$ & 328 \\
\hline $320 \mathrm{kHz}$ & $191 \mathrm{sec}$ & 4 & $8.9 \mathrm{e}-10$ & $9.6 \mathrm{e}-03$ & 656 \\
\hline $640 \mathrm{kHz}$ & $229 \mathrm{sec}$ & 5 & $4.2 \mathrm{e}-09$ & $1.9 \mathrm{e}-02$ & 1312 \\
\hline $1 \mathrm{MHz}$ & $316 \mathrm{sec}$ & 7 & $7.7 e-09$ & $3.0 \mathrm{e}-02$ & 2050 \\
\hline $5 \mathrm{MHz}$ & $1251 \mathrm{sec}$ & 30 & $1.4 \mathrm{e}-07$ & $1.5 \mathrm{e}-01$ & 10250 \\
\hline \multicolumn{6}{|c|}{$\begin{array}{l}\text { Preconditioner effectiveness is demonstrated over three decades of frequency. The residual is based on the } \\
\text { expression }\|\mathbf{K E}-\mathbf{S}\|^{2} /\|\mathbf{S}\|^{2} \text { Criteria for terminating the qmr iteration was based on a threshold value of } 1.0 \mathrm{e}-08 \\
\text { for the residual norm or a maximum of } 30 \text { iterations. Values based on equations }(16) \text { and (17) are also included } \\
\text { which aid in estimating when the preconditioner will be effective before the qmr solution is attempted. }\end{array}$} \\
\hline
\end{tabular}

In order to study the performance of the low frequency Neumann series expansion for multiple frequencies we first show run time statistics for the induction-logging problem, described above, when a total field, low frequency, preconditioned solution has been specified. Solution comparisons based on the total fields are needed because of the different types Neumann series solutions, we have implemented only the total field expansion. Also when a total field solution is employed it is necessary to drive the residual to a much lower level than with a scattered field solution, to insure accurate results. In Table 2 , we observe that the different solution approaches were terminated once the residual fell below a threshold value of 1e-13. As can be seen form the table, the series solution can compute responses for all frequencies faster that that of the preconditioner, which must be applied at each discrete frequency. What is interesting about these comparisons, however, is that the series solution did not perform as well as expected; we expected the series solution to solve the problem in an amount of time approximately equal to that required by the preconditioner at a discrete frequency. The reason for this discrepancy was that it was necessary to solve for each term in the series with far greater accuracy in equation (24), than what was needed in equations (10) and (12) at each preconditoned qmr iteration. 
TABLE 2

\begin{tabular}{|c|c|c|c|}
\hline \multicolumn{4}{|c|}{ Low Frequency Preconditioner } \\
\hline Frequency & Solution Time & qmr Iterations & Residual \\
\hline $10 \mathrm{kHz}$ & $185 \mathrm{sec}$ & 4 & $2.5 e-17$ \\
\hline $20 \mathrm{kHz}$ & $186 \mathrm{sec}$ & 4 & $2.5 \mathrm{e}-17$ \\
\hline $40 \mathrm{kHz}$ & $187 \mathrm{sec}$ & 4 & $4.1 \mathrm{e}-16$ \\
\hline $80 \mathrm{kHz}$ & $187 \mathrm{sec}$ & 4 & $1.2 \mathrm{e}-15$ \\
\hline $160 \mathrm{kHz}$ & $188 \mathrm{sec}$ & 4 & $4.6 \mathrm{e}-14$ \\
\hline Total Time & \multicolumn{3}{|l|}{935 seconds } \\
\hline \multicolumn{4}{|c|}{ Low frequency Neumann series expansion } \\
\hline Frequencies & Solution Time & Terms & Residual \\
\hline $10-160 \mathrm{kHz}$ & 570 seconds & 5 & $2.8 \mathrm{e}-15$ \\
\hline \multicolumn{4}{|c|}{$\begin{array}{l}\text { Comparison of solution time for the low frequency preconditioner and the Neumann series expansion at low } \\
\text { frequencies. A total field solution is assumed in both types of solution approaches. The total time required for the } \\
\text { preconditioner to compute solutions for all } 5 \text { frequencies is } 935 \text { seconds and is about a factor of two slower than } \\
\text { the series solution which computes responses at all frequencies simultaneously. The residual used in this } \\
\text { comparison to terminate both solutions was set at a threshold value of } 1.0 \mathrm{e}-13 \text {. }\end{array}$} \\
\hline
\end{tabular}

In Figure 1 we plot the convergence rate of the Neumann series solution at $640 \mathrm{kHz}$. Noticed that the series solution diverges after six terms, which contrast with the preconditioned solution, which converges at this frequency. This behavior is indicative of the robustness of the preconditioned Krylov method compared with a straight Neumann series solution approach and implies that the Lanczos method, which is a Krylov subspace technique (cf. Greenbaum, 1999), should be the optimal way for computing multiple frequency responses. 


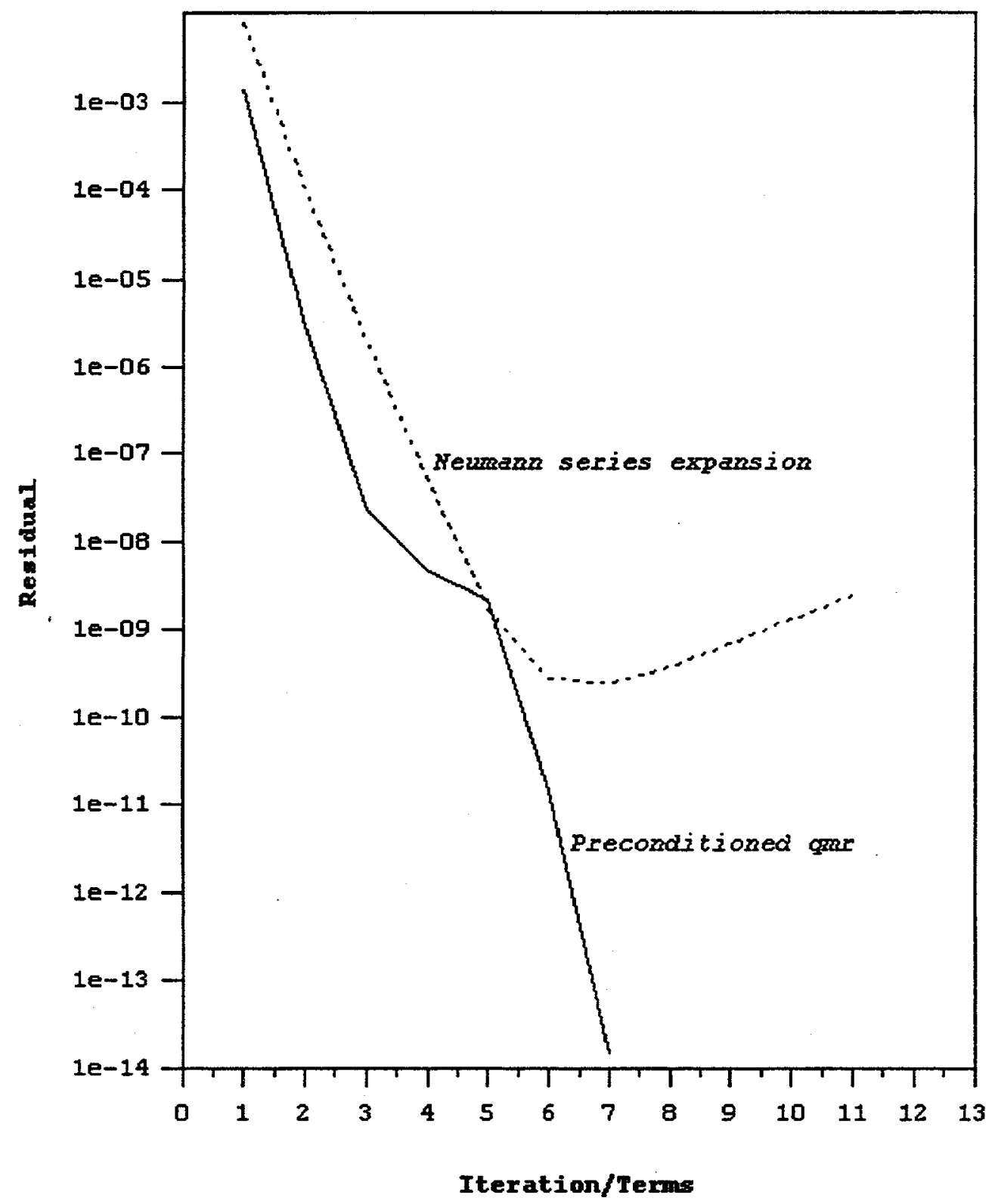

Figure 1. The plot shows the convergence behavior of the low frequency Neumann series expansion and preconditioner at $640 \mathrm{kHz}$. These results show the preconditioned solution to be far more robust than the series solution.

\section{CONCLUSIONS}

The techniques introduced in this report show good promise in accelerating the solutions of Maxwell's equations for subsurface imaging. The low frequency preconditioner has shown to be quite effective while the low frequency Neumann sereis expansion was to a lesser degree. Because of the premature termination of this project, it was not possible to implement all the procedures discussed in this report. Nevertheless, future work should focus on the implementation of spectral Lanczos methods, since such methods appear optimum for computing multiple frequency responses. 


\section{REFERENCES}

Alumbaugh, D. L., Newman, G. A, Prevost, L., and Shadid, J., 1996, Three-dimensional wideband electromagnetic modeling on massively parallel computers: Radio Sci., 31, 1-23.

Avdeev, D. B., Kuvshinov, A. V., Pankratov, O. V., Newman, G.A. and Alumbaugh D. L., 1999, Modeling induction log responses in deviated boreholes with anisotropic bedding: submitted to Geophysics

Druskin, V., Knizhnerman, L., and Lee P., 1999, A new spectral Lanczos decomposition method for induction modeling in arbitrary 3D geometry: Geophysics, 64, 701-706.

Druskin, V., Knizhnerman, L., 1989, Two polynomial methods of calculating functions of symmetric matricies: U. S. S. R. Comput. Maths. Math. Phys., 29, no. 6, 112-121.

Greenbaum, A., 1997, Iterative methods for solving linear systems, Society for Industrial and Applied Mathematics, Philadelphia.

Golub, G. H. and Van Loan, C. F., 1989, Matrix Computations, Johns Hopkins University Press, Baltimore.

Hohmann, G. W., and Ward, S. H, 1988, Electromagnetic theory for geophysical applications: Elelctromagnetic Methods, Volume I, Edited by M. N. Nabighian, Society of Exploration Geophysicists, Tulsa Ok.

LaBrecque, D., (1995), A scalar-vector potential solution for 3D EM finite difference modeling: Proceeding on the International Symposium in Three-dimensional Electromagnetics, Schlumberger-Doll Research, Ridgefield, Connecticut, 143-152.

Newman, G. A., and Alumbaugh, D. L., 1995, Frequency-domain modeling of airborne electromagnetic responses using staggered finite differences: Geophysical Prospecting, 43, 1021-1042.

Smith, J. T., 1996, Conservative modeling of 3-D electromagnetic fields; Part II: Biconjugate gradient solution and an accelertor: Geophysics, 61, 1319-1324.

Yee, K. S., 1966, Numerical solution of initial boundary value problems involving Maxwell's equations in isotropic media: IEEE Transactions on Antennas and Propagation, AP-14, 302-309. 


\section{APPENDIX}

Theorem. Let $\mathbf{A}^{+} \mathbf{S}_{\mathbf{0}}=\beta_{1} \mathbf{Q}_{m} \mathbf{e}_{1}$, after $m$ steps of a variant of the symmetric Lanczos algorithm that enforces $\mathbf{Q}_{m}{ }^{\mathbf{T}} \mathbf{A Q}_{m}=\mathbf{I}$ we have

$$
\left(\mathbf{A}^{+} \mathbf{D}\right)^{\mathrm{j}} \mathbf{A}^{+} \mathbf{S}_{\mathrm{o}}=\beta_{1} \mathbf{Q}_{m} \mathbf{T}_{m}^{\mathrm{j}} \mathbf{e}_{1, \quad \mathrm{j}=0,1,2, \ldots, m-1}
$$

Proof. First from Golub and Van Loan (1989) we can summarize $m$ steps of the symmetric Lanczos algorithm in equation (62) as

$$
\mathbf{L}^{-1} \mathbf{D} \mathbf{L}^{-\mathbf{T}} \mathbf{V}_{m}=\mathbf{V}_{m} \mathbf{T}_{m}+\beta_{m+1} \mathbf{V}_{m+1} \mathbf{e}_{m}^{\mathbf{T}}
$$

The vector $\mathbf{e}_{m}$ is the $m$-th column of the mxm identity matrix $\mathbf{I}_{m}$ When we multiply the above expression by $\mathbf{L}^{-\mathrm{T}}$ and use $\mathbf{Q}_{m}=\mathbf{L}^{-\mathrm{T}} \mathbf{V}_{m}$ and $\mathbf{L}^{-\mathrm{T}} \mathbf{L}^{-1}=\mathbf{A}^{+}$we then have

$$
\mathbf{A}^{+} \mathbf{D} \mathbf{Q}_{m}=\mathbf{Q}_{m} \mathbf{T}_{m}+\beta_{\mathrm{m}+1} \mathbf{L}^{-\mathbf{T}} \mathbf{v}_{\mathrm{m}+1} \mathbf{e}_{\mathrm{m}}^{\mathbf{T}}
$$

The rest of the proof follows that given by Druskin and Knizhnerman, (1989) and is by induction over $j$. For $\mathrm{j}=0$, we have

$$
\mathbf{A}^{+} \mathbf{S}_{\mathbf{o}}=\beta_{1} \mathbf{Q}_{m} \mathbf{e}_{1}
$$

And by the induction hypothesis we have that

$$
\begin{aligned}
\left(A^{+} D\right)^{j+1} A^{+} S_{o} & =\left(A^{+} D\right)\left(A^{+} D\right)^{j} A^{+} S_{\mathbf{v}} \\
& =\beta_{1}\left(A^{+} D\right) Q_{m} T_{m}^{j} \mathbf{e}_{1}=\beta_{1}\left(Q_{m} T_{m}+\beta_{m+1} L^{-T} \mathbf{v}_{m+1} \mathbf{e}_{m}\right) \mathbf{T}_{m}^{j} \mathbf{e}_{1} \\
& =\beta_{1} Q_{m} T_{m}{ }^{j+1} \mathbf{e}_{1}
\end{aligned}
$$

since $\mathbf{e}_{m}^{\mathbf{T}} \mathbf{T}_{m}^{\mathrm{j}} \mathbf{e}_{1}=0$, for $\mathrm{j} \leq m-2$ 


\section{Distribution}

\begin{tabular}{|c|c|}
\hline MS -0149 & Chuck Meyers, 4000 \\
\hline $\mathrm{MS}-0701$ & Les Shephard, 6100 \\
\hline MS-0750 & $\begin{array}{l}\text { David Aldridge, } 6116 \\
\text { Lewis Bartel, } 6116 \\
\text { Gregory Newman, } 6116(5) \\
\text { Neill Symons, } 6116 \\
\text { Marianne Walck, } 6116 \\
\text { Chester Weiss, } 6116\end{array}$ \\
\hline MS-1110 & $\begin{array}{l}\text { Dave Womble, } 9222 \\
\text { David Day, } 9222(5) \\
\text { Sue Minkoff, } 9222\end{array}$ \\
\hline MS-9018 & Central Technical Files, 8940-2 \\
\hline MS-0899 & Technical Library, 4916 (2) \\
\hline MS -0612 & Review \& Approval Desk, 4912 \\
\hline MS-0161 & Patent \& Licensing Office, 11500 \\
\hline
\end{tabular}

